\title{
Nonmetrizable topological dynamical characterization of central sets
}

\author{
by
}

\author{
Hong-ting $\mathrm{S} h$ i and Hong-wei Y ang (Beijing)
}

\begin{abstract}
Without the restriction of metrizability, topological dynamical systems $\left(X,\left\langle T_{s}\right\rangle_{s \in G}\right)$ are defined and uniform recurrence and proximality are studied. Some well known results are generalized and some new results are obtained. In particular, a topological dynamical characterization of central sets in an arbitrary semigroup $(G,+)$ is given and shown to be equivalent to the usual algebraic characterization.
\end{abstract}

0. Introduction. A topological dynamical system is usually defined to consist of a compact metric space $X$ together with a semigroup (or group) acting on $X$ by continuous transformations. (See [11, p. 19] or [2, Definition 6.1].) We generalize this notion by dropping the "metric" requirement and study in Section 1 natural generalizations of uniform recurrence and proximality. These generalizations turn out to be very useful, because they enable us to establish the equivalence of dynamical and algebraic characterizations of central sets.

The notion of "central subset" was first developed by Furstenberg [11] in the semigroup $(\mathbb{N},+)$ of natural numbers. Later Bergelson and Hindman [2] defined the notion of a central set in an arbitrary semigroup $(G,+)$ in terms of the algebra of $\beta G$, the Stone-Cech compactification of $G$. They also defined a notion of *-central subset, using the natural extension of Furstenberg's definition, and pointed out that any *-central subset of $(G,+)$ is central in $(G,+)$. Moreover, a result of Weiss (see [2, Theorem 6.11]) guarantees that in a countable semigroup $(G,+)$, a subset of $G$ is central if and only if it is *-central. In Section 2 of this paper, we show (inspired by Weiss) that if ${ }^{* *}$-central is defined as the natural extension of the notion of *-central using the more general definition of topological dynamical system,

1991 Mathematics Subject Classification: 54H20, 22A15.

Key words and phrases: topological dynamical system, enveloping semigroup, uniform recurrence, proximality, minimal idempotent, central subset. 
then in any semigroup, countable or not, a subset is central if and only if it is $* *$-central.

Throughout this paper, $(G,+)$ will denote an infinite discrete semigroup and $(\beta G,+)$ will denote its Stone-Cech compactification with the operation extending the operation in $G$ such that $\lambda_{u}$ is continuous for each $u \in \beta G$ and $\varrho_{s}$ is continuous for each $s \in G$, where for $v \in \beta G$ we define $\lambda_{u}(v)=u+v$ and $\varrho_{s}(v)=v+s$. Then $(\beta G,+)$ is a compact left topological semigroup. As such, $(\beta G,+)$ has a unique smallest two-sided ideal which is the union of all minimal right ideals of $(\beta G,+)$ and is also the union of all minimal left ideals of $(\beta G,+)$. (See [4, Theorem 1.3.11].) We will also use the fact that any compact left topological semigroup has an idempotent $[8$, Corollary 2.10].

From [12] we know that for each point $u \in \beta G$, there is a unique ultrafilter on $G$ which converges to $u$. Following [12] we will denote this ultrafilter by $A^{u}$. Given $u, v \in \beta G$ and $B \subseteq G$ one has $B \in A^{u+v}$ if and only if $\left\{s \in G: B-s \in A^{u}\right\} \in A^{v}$, where $B-s=\{t \in G: t+s \in B\}$. (See [13].)

1. Uniform recurrence and proximality. We begin by generalizing the notion of topological dynamical system to apply to an arbitrary compact space.

Definition 1.1. A topological dynamical system is a pair $\left(X,\left\langle T_{s}\right\rangle_{s \in G}\right)$, where $X$ is a compact Hausdorff space, $(G,+)$ is an infinite discrete semigroup, each $T_{s}$ is a continuous mapping from $X$ to $X$, and $T_{s} \circ T_{t}=T_{t+s}$ for each $s, t \in G$.

We note that one can apply the same definition to an arbitrary completely regular Hausdorff space $X$. But one gains no generality by doing so since then $\left(X,\left\langle T_{s}\right\rangle_{s \in G}\right)$ is a topological dynamical system if and only if $\left(\beta X,\left\langle T_{s}^{\beta}\right\rangle_{s \in G}\right)$ is a topological dynamical system.

We now recall the following definition from [10].

Definition 1.2. Let $X$ be a topological space, let $D$ be a discrete space, let $u \in \beta D$, and for each $s \in D$, let $x_{s} \in X$. Then for $y \in X, y=u-\lim _{s \in D} x_{s}$ if and only if for each neighborhood $U$ of $y,\left\{s \in D: x_{s} \in U\right\} \in A^{u}$.

It is well known and easy to see that in a compact Hausdorff space $X$ there is always a unique $u$ - $\lim _{s \in D} x_{s}$.

We recall from $[6,7,8]$ the notion of an enveloping semigroup.

Definition 1.3. Let $X$ be a compact Hausdorff space, let $\prod_{x \in X} X$ have the product topology, and let $\mathcal{S}$ be a set of continuous functions from $X$ to $X$. The enveloping semigroup $E(\mathcal{S})$ of $\mathcal{S}$ is the closure of $\mathcal{S}$ in $\prod_{x \in X} X$.

We now extend the family $\left\langle T_{s}\right\rangle_{s \in G}$ by defining $T_{u}$ for $u \in \beta G$. 
Definition 1.4. Let $\left(X,\left\langle T_{s}\right\rangle_{s \in G}\right)$ be a topological dynamical system and let $u \in \beta G$. Then $T_{u}=u$ - $\lim _{s \in G} T_{s}$, where the limit is taken in $\prod_{x \in X} X$.

Note that if $t \in G$, then $T_{t}=t-\lim _{s \in G} T_{s}$ so the above definition is consistent with the earlier notion.

We have immediately the following characterization of $E\left(\left\langle T_{s}\right\rangle_{s \in G}\right)$.

Theorem 1.5. Let $\left(X,\left\langle T_{s}\right\rangle_{s \in G}\right)$ be a topological dynamical system. Then $E\left(\left\langle T_{s}\right\rangle_{s \in G}\right)=\left\{T_{u}: u \in \beta G\right\}$.

Proof. Define $\varphi: G \rightarrow \prod_{x \in X} X$ by $\varphi(s)=T_{s}$ and let $\varphi^{\beta}$ be the continuous extension to $\beta G$. Then by [5, Lemma 2.1], for each $u \in \beta G$, $\varphi^{\beta}(u)=T_{u}$. Thus $\left\{T_{u}: u \in \beta G\right\}=\varphi^{\beta}[\beta G]$ is a closed subset of $\prod_{x \in X} X$ containing $\left\{T_{s}: s \in G\right\}$. Consequently, $E\left(\left\langle T_{s}\right\rangle_{s \in G}\right) \subseteq\left\{T_{u}: u \in \beta G\right\}$.

On the other hand, each $T_{u}=u-\lim _{s \in G} T_{s} \in \operatorname{cl}\left\{T_{s}: s \in G\right\}$ so $E\left(\left\langle T_{s}\right\rangle_{s \in G}\right)$ $=\left\{T_{u}: u \in \beta G\right\}$.

The following observation will frequently be useful.

Lemma 1.6. Let $\left(X,\left\langle T_{s}\right\rangle_{s \in G}\right)$ be a topological dynamical system and let $u, v \in \beta G$. Then $T_{u} \circ T_{v}=T_{v+u}$.

Pr o o f. Let $x \in X$. Then by [2, Lemma 6.10$]$,

$$
\begin{aligned}
T_{u+v}(x) & =(u+v)-\lim _{r \in G} T_{r}(x)=v-\lim _{t \in G}\left(u-\lim _{s \in G} T_{s+t}(x)\right) \\
& =v-\lim _{t \in G}\left(T_{t}\left(u-\lim _{s \in G} T_{s}(x)\right)\right)=v-\lim _{t \in G}\left(T_{t}\left(T_{u}(x)\right)\right)=T_{v}\left(T_{u}(x)\right) .
\end{aligned}
$$

We now extend the notion of uniform recurrence to apply to an arbitrary topological dynamical system (using the usual definition). We also extend the standard notion of minimal closed invariant sets.

Definition 1.7. Let $\left(X,\left\langle T_{s}\right\rangle_{s \in G}\right)$ be a topological dynamical system.

(a) A subset $D$ of $G$ is called syndetic if and only if there exists a finite subset $F$ of $G$ such that for any $s \in G$, there exists $t \in F$ with $s+t \in D$.

(b) A point $x \in X$ is a uniformly recurrent point if and only if for each neighborhood $W$ of $x,\left\{s \in G: T_{s}(x) \in W\right\}$ is syndetic.

(c) A subset $R$ of $X$ is invariant if and only if for each $s \in G, T_{s}[R] \subseteq R$.

(d) A subset $R$ of $X$ is a minimal closed invariant subset if and only if $R$ is minimal in the set $\{P \subseteq X: P$ is nonempty, closed, and invariant $\}$.

The following lemma generalizes [11, Theorems 1.15 and 1.17]. The proof is the same as in [11], so we omit it.

Lemma 1.8. Let $\left(X,\left\langle T_{s}\right\rangle_{s \in G}\right)$ be a topological dynamical system.

(a) If $R$ is a minimal closed invariant subset of $X$, then each point of $R$ is uniformly recurrent. 
(b) If $x$ is a uniformly recurrent point, then $\operatorname{cl}\left\{T_{s}(x): s \in G\right\}$ is a minimal closed invariant subset of $X$ and $x \in \operatorname{cl}\left\{T_{s}(x): s \in G\right\}$.

Note that $\left(\beta G,\left\langle\varrho_{s}\right\rangle_{s \in G}\right)$ is a topological dynamical system.

Theorem 1.9. Let $R \subseteq \beta G$. Then $R$ is a minimal closed invariant subset of the topological dynamical system $\left(\beta G,\left\langle\varrho_{s}\right\rangle_{s \in G}\right)$ if and only if $R$ is a minimal right ideal of the semigroup $(\beta G,+)$.

P r o of. Note first that any right ideal of $(\beta G,+)$ is invariant. Conversely any closed invariant subset $P$ of $\beta G$ is a right ideal of $(\beta G,+)$. (To verify the latter assertion, note that for each $u \in P$ and each $s \in G, u+s \in P$. Consequently, given $u \in P, u+\beta G=\lambda_{u}[\operatorname{cl} G] \subseteq \operatorname{cl}\left(\lambda_{u}[G]\right) \subseteq \operatorname{cl} P=P$.)

Assume now that $R$ is a minimal closed invariant subset of the topological dynamical system $\left(\beta G,\left\langle\varrho_{s}\right\rangle_{s \in G}\right)$. Then $R$ is a right ideal of $(\beta G,+)$. Choose a minimal right ideal $R^{\prime}$ of $(\beta G,+)$ which is contained in $R$. Since minimal right ideals are closed [4, Theorem 1.3.11], $R^{\prime}$ is a closed invariant subset of $R$ and hence $R^{\prime}=R$.

Similarly, if $R$ is a minimal right ideal of $(\beta G,+)$, then $R$ is closed and invariant while any closed invariant subset of $R$ is a right ideal, and hence $R$ is a minimal closed invariant set.

COROLlaRY 1.10. The set of all uniformly recurrent points of the topological dynamical system $\left(\beta G,\left\langle\varrho_{s}\right\rangle_{s \in G}\right)$ is the smallest two-sided ideal of $(\beta G,+)$.

Proof. This is an immediate consequence of Lemma 1.8, Theorem 1.9, and the fact that the smallest ideal of $(\beta G,+)$ is the union of all the minimal right ideals of $(\beta G,+)$.

The following theorem is a generalization of [2, Lemmas 6.6 and 6.9] and [13, Theorem 6.2]. This theorem is the theoretical basis of Theorem 2.4.

TheOREM 1.11. Let $\left(X,\left\langle T_{s}\right\rangle_{s \in G}\right)$ be a topological dynamical system, let $R$ be a minimal right ideal of $(\beta G,+)$ and let $x \in X$. The following statements are equivalent.

(a) The point $x$ is a uniformly recurrent point of $\left(X,\left\langle T_{s}\right\rangle_{s \in G}\right)$.

(b) There exists $u \in R$ such that $T_{u}(x)=x$.

(c) There exists $y \in X$ and an idempotent $u \in R$ such that $T_{u}(y)=x$.

(d) There exists an idempotent $u \in R$ such that $T_{u}(x)=x$.

Proof. (a) $\Rightarrow$ (b). Choose any $v \in R$. Let $\mathcal{N}$ be the set of neighborhoods of $x$ in $X$. For each $U \in \mathcal{N}$ let $B_{U}=\left\{s \in G: T_{s}(x) \in U\right\}$. Since $x$ is uniformly recurrent, each $B_{U}$ is syndetic, so choose finite $F_{U} \subseteq G$ such that for any $s \in G$ there is some $t \in F_{U}$ such that $s+t \in B_{U}$. Then for each $U \in \mathcal{N}$, we have $G=\bigcup_{t \in F_{U}}\left(B_{U}-t\right)$; so pick $t_{U} \in F_{U}$ such that $B_{U}-t_{U} \in A^{v}$. 
Given any $U \in \mathcal{N}$, let $C_{U}=\left\{t_{V}: V \in \mathcal{N}\right.$ and $\left.V \subseteq U\right\}$. Then $\left\{C_{U}:\right.$ $U \in \mathcal{N}\}$ has the finite intersection property so pick $w \in \beta G$ such that $\left\{C_{U}: U \in \mathcal{N}\right\} \subseteq A^{w}$ and let $u=v+w$. Then $u \in R$ since $R$ is a right ideal of $(\beta G,+)$. To see that $T_{u}(x)=x$, let $U \in \mathcal{N}$. To see that $B_{U} \in A^{u}$, suppose instead that $B_{U} \notin A^{u}$. Then $\left\{t \in G: B_{U}-t \notin A^{v}\right\} \in A^{w}$ and $C_{U} \in A^{w}$ so pick $t \in C_{U}$ such that $B_{U}-t \notin A^{v}$. Pick $V \in \mathcal{N}$ with $V \subseteq U$ such that $t=t_{V}$. Then $B_{V}-t \in A^{v}$ and $B_{V}-t \subseteq B_{U}-t$, a contradiction.

(b) $\Rightarrow$ (c). Let $S=\left\{v \in R: T_{v}(x)=x\right\}$. It suffices to show that $S$ is a compact subsemigroup of $R$, since then $S$ has an idempotent. By assumption, $S \neq \emptyset$. Further, if $v \in R \backslash S$, then there is some neighborhood $U$ of $x$ such that $B=\left\{s \in G: T_{s}(x) \in U\right\} \notin A^{v}$. Then $\mathrm{cl} B$ is a neighborhood of $v$ in $\beta G$ which misses $S$, and so $S$ is compact. Finally, to see that $S$ is a semigroup, let $v, w \in S$. Then by Lemma 1.6, $T_{v+w}(x)=T_{w}\left(T_{v}(x)\right)=T_{w}(x)=x$.

(c) $\Rightarrow(\mathrm{d})$. Again we use Lemma 1.6: $T_{u}(x)=T_{u}\left(T_{u}(y)\right)=T_{u+u}(y)=$ $T_{u}(y)=x$.

$(\mathrm{d}) \Rightarrow\left(\right.$ a). Let $U$ be a neighborhood of $x$ and let $B=\left\{s \in G: T_{s}(x) \in U\right\}$ and suppose that $B$ is not syndetic. Then

$$
\left\{G \backslash \bigcup_{t \in F} B-t: F \text { is a finite nonempty subset of } G\right\}
$$

has the finite intersection property, so pick some $w \in \beta G$ such that

$$
\left\{G \backslash \bigcup_{t \in F} B-t: F \text { is a finite nonempty subset of } G\right\} \subseteq A^{w} .
$$

Then $(w+\beta G) \cap \operatorname{cl} B=\emptyset$. (For suppose instead one had some $v \in \beta G$ with $B \in A^{w+v}$. Then pick some $t \in G$ with $B-t \in A^{w}$.) Let $R^{\prime}=w+\beta G$. Then $R^{\prime}$ is a right ideal of $(\beta G,+)$, so $u+R^{\prime}$ is a right ideal of $(\beta G,+)$ which is contained in $R$, and hence $u+R^{\prime}=R$. Thus we may pick some $v \in R^{\prime}$ such that $u+v=u$. Again using Lemma 1.6, $T_{v}(x)=T_{v}\left(T_{u}(x)\right)=$ $T_{u+v}(x)=T_{u}(x)=x$, so in particular $B \in A^{v}$. But $v \in R^{\prime}$ and $R^{\prime} \cap \mathrm{cl} B=\emptyset$, a contradiction.

The previous generalizations have all been straightforward. It is not so clear how to generalize the notion of "proximal". Recall that in a metric dynamical system $\left(X,\left\langle T_{s}\right\rangle_{s \in G}\right)$, two points $x$ and $y$ are proximal provided there is a sequence $\left\langle s_{k}\right\rangle_{k=1}^{\infty}$ in $G$ such that $\lim _{k \rightarrow \infty} d\left(T_{s_{k}}(x), T_{s_{k}}(y)\right)=0$, where $d$ is the metric on $X$.

DeFinition 1.12. Let $\left(X,\left\langle T_{s}\right\rangle_{s \in G}\right)$ be a topological dynamical system and let $x, y \in X$. Then $x$ and $y$ are proximal if and only if there is some $u \in \beta G$ such that $T_{u}(x)=T_{u}(y)$.

We show first that we have indeed generalized the metric notion. 
TheOREM 1.13. Let $\left(X,\left\langle T_{s}\right\rangle_{s \in G}\right)$ be a topological dynamical system, let $x, y \in X$, and assume that $X$ is a metric space with metric $d$. Then $x$ and $y$ are proximal if and only if there is a sequence $\left\langle s_{k}\right\rangle_{k=1}^{\infty}$ in $G$ such that $\lim _{k \rightarrow \infty} d\left(T_{s_{k}}(x), T_{s_{k}}(y)\right)=0$.

Proof. Assume we have $u \in \beta G$ such that $T_{u}(x)=T_{u}(y)=z$. Then for each $k \in \mathbb{N}, B_{k}=\left\{s \in G: d\left(T_{s}(x), z\right)<1 / k\right\} \cap\left\{s \in G: d\left(T_{s}(y), z\right)<\right.$ $1 / k\} \in A^{u}$, so choose $s_{k} \in B_{k}$. Then $\lim _{k \rightarrow \infty} d\left(T_{s_{k}}(x), T_{s_{k}}(y)\right)=0$.

Now assume that we have a sequence $\left\langle s_{k}\right\rangle_{k=1}^{\infty}$ in $G$ such that $\lim _{k \rightarrow \infty} d\left(T_{s_{k}}(x), T_{s_{k}}(y)\right)=0$. Choose $u \in \beta G$ such that $\left\{\left\{s_{n}: n \geq k\right\}\right.$ : $k \in \mathbb{N}\} \subseteq A^{u}$. Let $z=T_{u}(x)$. To see that $z=T_{u}(y)$, let $\varepsilon>0$ be given and let $B=\left\{s \in G: d\left(T_{s}(x), z\right)<\varepsilon / 2\right\}$. Then $B \in A^{u}$. Pick $k \in \mathbb{N}$ such that for all $n>k, d\left(T_{s_{n}}(x), T_{s_{n}}(y)\right)<\varepsilon / 2$ and let $C=\left\{s_{n}: n \geq k\right\}$. Then $B \cap C \in A^{u}$ and $B \cap C \subseteq\left\{s \in G: d\left(T_{s}(y), z\right)<\varepsilon\right\}$.

The following theorem is a generalization of [11,Theorem 8.7].

THEOREM 1.14. Let $\left(X,\left\langle T_{s}\right\rangle_{s \in G}\right)$ be a topological dynamical system and let $x \in X$. Then there is a uniformly recurrent point $y \in \operatorname{cl}\left\{T_{s}(x): s \in G\right\}$ such that $x$ and $y$ are proximal.

Proof. Let $R$ be any minimal right ideal of $(\beta G,+)$ and pick an idempotent $u \in R$. Let $y=T_{u}(x)$. Then trivially $y \in \operatorname{cl}\left\{T_{s}(x): s \in G\right\}$. By Theorem 1.11, $y$ is a uniformly recurrent point of $\left(X,\left\langle T_{s}\right\rangle_{s \in G}\right)$. By Lemma 1.6 we have $T_{u}(y)=T_{u}\left(T_{u}(x)\right)=T_{u+u}(x)=T_{u}(x)$ so $x$ and $y$ are proximal.

The following theorem is a generalization of [2, Lemma 6.4], which was itself a generalization of [8, Lemma 5.15].

THeOREM 1.15. Let $\left(X,\left\langle T_{s}\right\rangle_{s \in G}\right)$ be a topological dynamical system and let $x, y \in X$. If $x$ and $y$ are proximal, then there is a minimal right ideal $R$ of $(\beta G,+)$ such that $T_{u}(x)=T_{u}(y)$ for all $u \in R$.

Proof. Pick $v \in \beta G$ such that $T_{v}(x)=T_{v}(y)$ and pick a minimal right ideal $R$ of $(\beta G,+)$ such that $R \subseteq v+\beta G$. To see that $R$ is as required, let $u \in R$ and choose $w \in \beta G$ such that $u=v+w$. Then, again using Lemma 1.6, we have $T_{u}(x)=T_{v+w}(x)=T_{w}\left(T_{v}(x)\right)=T_{w}\left(T_{v}(y)\right)=T_{v+w}(y)=T_{u}(y)$.

2. Central, *-central, and **-central sets. Recall that an idempotent $u$ in a compact left topological semigroup $(S,+)$ is said to be minimal if and only if $u$ is a member of the smallest ideal of $(S,+)$. Of course, since the smallest ideal is the union of all of the minimal right ideals, this is the same as saying that $u$ is a member of some minimal right ideal of $(S,+)$. Less obvious is that this is equivalent to being minimal with respect to the ordering of idempotents which has $u \leq v$ if and only if $u+v=v+u=u$. 
(See [4] or [2, Lemma 3.2] for a proof of this equivalence.) We also recall the following definitions from [2].

DeFinition 2.1. Let $(G,+)$ be a discrete semigroup.

(a) A subset $B$ of $G$ is central if and only if there is a minimal idempotent $u$ of $(\beta G,+)$ such that $B \in A^{u}$.

(b) A subset $B$ of $G$ is ${ }^{*}$-central if and only if there exists a topological dynamical system $\left(X,\left\langle T_{s}\right\rangle_{s \in G}\right)$ such that $X$ is a (compact) metric space and there exist $x, y \in X$ and a neighborhood $U$ of $y$ such that $x$ and $y$ are proximal, $y$ is uniformly recurrent, and $B=\left\{s \in G: T_{s}(x) \in U\right\}$.

We extend the notion of *-central by dropping the requirement that $X$ be a metric space. Note that the fact which allows us to do this is that we were able to extend the notion of "proximal" to the nonmetric situation.

Definition 2.2. Let $(G,+)$ be a discrete semigroup. A subset $B$ of $G$ is ${ }^{* *}$-central if and only if there exists a topological dynamical system $\left(X,\left\langle T_{s}\right\rangle_{s \in G}\right)$ and there exist $x, y \in X$ and a neighborhood $U$ of $y$ such that $x$ and $y$ are proximal, $y$ is uniformly recurrent, and $B=\left\{s \in G: T_{s}(x) \in U\right\}$.

We have the following easy consequence of the results in Section 1.

THEOREM 2.3. Let $\left(X,\left\langle T_{s}\right\rangle_{s \in G}\right)$ be a topological dynamical system and let $x, y \in X$. There is a minimal idempotent $u$ in $(\beta G,+)$ such that $T_{u}(x)=y$ if and only if both $y$ is uniformly recurrent and $x$ and $y$ are proximal.

Proof. $(\Rightarrow)$. Since $u$ is minimal, there is a minimal right ideal $R$ of $(\beta G,+)$ such that $u \in R$. Thus by Theorem 1.11, $y$ is uniformly recurrent. By Lemma 1.6, $T_{u}(y)=T_{u}\left(T_{u}(x)\right)=T_{u+u}(x)=T_{u}(x)$ so $x$ and $y$ are proximal.

$(\Leftarrow)$. Pick by Theorem 1.15 a minimal right ideal $R$ of $(\beta G,+)$ such that $T_{u}(x)=T_{u}(y)$ for all $u \in R$. Pick by Theorem 1.11 an idempotent $u \in R$ such that $T_{u}(y)=y$.

The following is the main result of this paper. The proof of the necessity is inspired by the result of Weiss [2, Theorem 6.11].

Theorem 2.4. Let $(G,+)$ be an infinite discrete semigroup and let $B \subseteq$ $G$. Then $B$ is central if and only if $B$ is **-central.

Proof. $(\Rightarrow)$. We may assume $G$ has an identity 0 . (If not, adjoin one, in which case $0 \notin B$.) Let $X=\prod_{s \in G}\{0,1\}$ and for $s \in G$ define $T_{s}: X \rightarrow X$ by $T_{s}(x)(t)=x(s+t)$. It is routine to verify that each $T_{s}$ is continuous. Now let $x=\chi_{B}$, the characteristic function of $B$. That is, $x(t)=1$ if and only if $t \in B$. Pick a minimal $u$ in $(\beta G,+)$ such that $B \in A^{u}$ and let $y=T_{u}(x)$. Then by Theorem 2.3, $y$ is uniformly recurrent and $x$ and $y$ are proximal. 
Now let $U=\{z \in X: z(0)=y(0)\}$. Then $U$ is a neighborhood of $y$ in $X$. We note that $y(0)=1$. Indeed, $y=T_{u}(x)$ so $\left\{s \in G: T_{s}(x) \in U\right\} \in A^{u}$ and we may choose some $s \in B$ such that $T_{s}(x) \in U$. Then $y(0)=T_{s}(x)(0)=$ $x(s+0)=1$. Thus given any $s \in G$,

$$
s \in B \Leftrightarrow x(s)=1 \Leftrightarrow T_{s}(x)(0)=1 \Leftrightarrow T_{s}(x) \in U .
$$

$(\Leftarrow)$. Choose a topological dynamical system $\left(X,\left\langle T_{s}\right\rangle_{s \in G}\right)$, points $x, y \in$ $X$, and a neighborhood $U$ of $y$ such that $x$ and $y$ are proximal, $y$ is uniformly recurrent, and $B=\left\{s \in G: T_{s}(x) \in U\right\}$. Choose by Theorem 2.3 a minimal idempotent $u$ in $(\beta G,+)$ such that $T_{u}(x)=y$. Then $B \in A^{u}$.

As was the situation with *-central sets, it is not obvious that the notion of $* *$-central is closed under supersets.

Corollary 2.5. Let $(G,+)$ be an infinite discrete semigroup and let $B \subseteq C \subseteq G$. If $B$ is **-central, then $C$ is ${ }^{* *}$-central.

Proof. This follows from Theorem 2.4 and the fact that supersets of central sets are central.

In most cases a given topological dynamical system $\left(X,\left\langle T_{s}\right\rangle_{s \in G}\right)$ will determine infinitely many ${ }^{* *}$-central sets. We close with an interesting class of exceptions. (Note that the hypothesis of Theorem 2.6 is satisfied if one has no $s, t \in G$ with $t=t+s$.)

Theorem 2.6. Let $(G,+)$ be an infinite discrete semigroup and assume that for any $t \in G$ the set $\{s \in G: t=t+s\}$ is not syndetic. Then there is a topological dynamical system $\left(X,\left\langle T_{s}\right\rangle_{s \in G}\right)$ such that the only ${ }^{* *}$-central subset of $G$ determined by $\left(X,\left\langle T_{s}\right\rangle_{s \in G}\right)$ is $G$.

Pro of. Let $X$ be the hedgehog space of spininess $|G|$. (See [9].) That is, the set $X$ is the quotient of the set $[0,1] \times G$ obtained by collapsing $\{0\} \times G$ to a point, which we will call $\overline{0}$. Define a metric on $X$ by $\varrho(\overline{0},(x, t))=x$ and

$$
\varrho((x, s),(y, t))= \begin{cases}|x-y| & \text { if } s=t \\ x+y & \text { if } s \neq t .\end{cases}
$$

It is routine to verify that $\varrho$ is a metric on $X$.

Given $s \in G$ define $T_{s}: X \rightarrow X$ by $T_{s}(\overline{0})=\overline{0}$ and $T_{s}(x, t)=(x, t+s)$. It is routine to verify that each $T_{s}$ is continuous. Note also that the only uniformly recurrent point of $\left(X,\left\langle T_{s}\right\rangle_{s \in G}\right)$ is $\overline{0}$. To see this, suppose that $(x, t) \in(0,1] \times G$ is uniformly recurrent and let $U$ be the open ball of radius $x$ around $(x, t)$, that is,

$$
U= \begin{cases}\{(y, t): 0<y<2 x\} & \text { if } 0<x \leq 1 / 2, \\ \{(y, t): 0<y \leq 1\} & \text { if } 1 / 2<x \leq 1\end{cases}
$$

Then $\left\{s \in G: T_{s}(x, t) \in U\right\}=\{s \in G: t=t+s\}$, which is not syndetic by hypothesis. 
Further, the only point of $X$ which is proximal to $\overline{0}$ is $\overline{0}$ itself. Given any neighborhood $W$ of $\overline{0}, G=\left\{s \in G: T_{s}(\overline{0}) \in W\right\}$.

Acknowledgements. The manuscript of this paper was completed while the first author was visiting Peking University during the 1993-94 academic year. We would like to express our sincere thanks to Professor Zhang Zhifen, Professor Tanyun and Dr. Qi Dongwen for many helpful discussions and suggestions. We are particularly grateful to the referee for providing an improved version of the original paper.

\section{References}

[1] J. Auslander, Minimal Flows and their Extensions, North-Holland, Amsterdam, 1988.

[2] V. Bergelson and N. Hindman, Nonmetrizable topological dynamics and Ramsey theory, Trans. Amer. Math. Soc. 320 (1990), 293-320.

[3] J. Berglund and N. Hindman, Filters and the weak almost periodic compactification of a discrete semigroup, ibid. 284 (1984), 1-38.

[4] J. Berglund, H. Junghenn and P. Milnes, Analysis on Semigroups, Wiley, New York, 1989.

[5] E. van Douwen, The Čech-Stone compactification of a discrete groupoid, Topology Appl. 39 (1991), 43-60.

[6] R. Ellis, A semigroup associated with a transformation group, Trans. Amer. Math. Soc. 94 (1960), 272-281.

[7] —, Locally compact transformation groups, Duke Math. J. 24 (1957), 119-125.

[8] —, Lectures on Topological Dynamics, Benjamin, New York, 1969.

[9] R. Engelking, General Topology, Polish Scientific Publishers, Warszawa, 1977.

[10] Z. Frolík, Sums of ultrafilters, Bull. Amer. Math. Soc. 73 (1967), 87-91.

[11] H. Furstenberg, Recurrence in Ergodic Theory and Combinatorical Number Theory, Princeton University Press, Princeton, 1981.

[12] L. Gillman and M. Jerison, Rings of Continuous Functions, van Nostrand, Princeton, 1960.

[13] N. Hindman, Ultrafilters and Ramsey Theory - an update, in: Set Theory and its Applications, J. Steprāns and S. Watson (eds.), Lecture Notes in Math. 1401, Springer, 1989, 97-118.

Department of Mathematics

Capital Normal University

Beijing, 00037, P. R. China

Received 16 March 1995;

in revised form 21 November 1995 